\title{
Surface properties of calcium carbonate modified with silica by sol-gel method
}

Jorge Pedrosa, J.A.F. Gamelas*, Ana F. Lourenço, Paulo J. Ferreira

Chemical Engineering Department, CIEPQPF, University of Coimbra, Pólo II. R. Sílvio Lima, PT - 3030-790 Coimbra, Portugal

* jafgas@ eq.uc.pt; Tel: 00351239798740; fax: 00351239798703

\begin{abstract}
The physico-chemical surface properties of precipitated calcium carbonate (PCC) after modification with different amounts of silica by the sol-gel method were evaluated and compared to those of the original PCC, using inverse gas chromatography. The modification of the surface of PCC crystals by a dense film of silica provided a slight decrease of the dispersive component of the surface energy and a significant decrease of the specific component of the work of adhesion with trichloromethane (a Lewis acidic probe). In addition, interactions measured with weak Lewis bases (1-alkenes) also revealed differences between PCC and PCC-silica samples. Differences in the surface properties (namely of the Lewis acid-base ones) of the PCCs modified with distinct silica content (ca. 22 and $30 \mathrm{wt} \%$ ), were also found, and were interpreted as being due to the different accessibility of silanol groups $(\mathrm{Si}-\mathrm{OH})$ on the materials surface: a higher prevalence of these acidic groups in the material with lower silica content increases the specific affinity to Lewis basic probes and decreases the affinity to Lewis acidic probe. X-ray photoelectron spectroscopy results confirmed the presence of silica on PCC, with a higher Si/Ca atomic ratio being found for the material with higher silica content.
\end{abstract}

Keywords: Precipitated calcium carbonate; silica; sol-gel method; surface physicalchemical properties; IGC; XPS 


\section{Introduction}

Calcium carbonate is one of the most common fillers used in composite materials such as plastics and paper. Particularly, when used for the production of paper handsheets, cellulosic fibres are partially substituted by calcium carbonate (usually, as ground calcium carbonate (GCC) or as precipitated calcium carbonate (PCC)), reducing highly the costs of paper production and imparting good properties to the sheets, such as brightness, opacity or bulk. However, it reduces drastically the paper strength properties, especially at high filler levels. Thus, among other strategies, efforts have been made to modify the calcium carbonate surface in order to overcome its reduced potential to bind with fibres [1-3]. Recently, modification of the PCC surface with silica produced by sol-gel method has been reported. The PCC surface was modified with silica produced via hydrolysis and condensation reactions of tetraethylorthosilicate (silica precursor) in ethanol/water solutions under alkaline conditions. By varying the experimental conditions, namely the ammonia concentration of the reaction medium, materials with different silica contents were produced [4]. When the new hybrids were applied as fillers in papermaking an improvement of the paper strength properties was detected [5-6]. It is therefore of utmost importance to fully characterize the modified PCC particles for their bulk and surface properties in order to get a better understanding of their behaviour relatively to the unmodified PCC. The characterization of the fillers for their surface chemical properties is also highly relevant for anticipating their interactions with polymeric matrices other than fibres, such as poly-vinyl alcohol, polylactic acid, polyethylene, in the production of composite structures.

Inverse gas chromatography (IGC) is recognised to be an appropriate tool to evaluate the surface properties of powdered materials such as calcium carbonate. This 
technique enables obtaining a wide range of physicochemical surface parameters of the material under analysis not accessed by other techniques. The dispersive component of the surface energy $\left(\gamma_{s}^{d}\right)$, the Lewis acid-base character of the surface, nanoroughness parameters or specific interaction parameters with other components regarding the production of composite structures, can be assessed [7-10]. Several papers have reported the evaluation of the surface properties of calcium carbonates by inverse gas chromatography [11-22]. The dispersive component of the surface energy has been determined, and, values (at $100^{\circ} \mathrm{C}$ ) as low as ca. $30 \mathrm{~mJ} \mathrm{~m}^{-2}[18]$ or as high as $250 \mathrm{~mJ} \mathrm{~m}^{-}$ ${ }^{2}$ [17] have been reported. Most of the values, however, fall in the $40-70 \mathrm{~mJ} \mathrm{~m}^{-2}$ range $[12,15,16,19,20,22]$. Particularly, Keller and Luner [17] determined, at $100{ }^{\circ} \mathrm{C}, \gamma_{\mathrm{s}}^{\mathrm{d}}$ values of ca. $250 \mathrm{~mJ} \mathrm{~m}^{-2}$ and $56 \mathrm{~mJ} \mathrm{~m}^{-2}$ for precipitated calcium carbonate preconditioned, respectively, at $300{ }^{\circ} \mathrm{C}$ and $100{ }^{\circ} \mathrm{C}$. The apparent contradictory values reported in several papers can largely been explained by the effect of different amounts of physisorbed water at the calcium carbonate surfaces. It was proposed that by heating PCC at certain temperatures, the adsorbed surface water layer is removed, then exposing highly polar sites for the interaction with $n$-alkanes (mainly by dispersion (London) and induction (Debye) forces), thus increasing the dispersive component of the surface energy of the material [17]. Typically, higher $\gamma_{\mathrm{s}}^{\mathrm{d}}$ values are thus obtained for "more dried" calcium carbonate samples, that is, for samples pre-conditioned and analysed at higher temperatures. Besides the conditioning (and analysis) temperature, the origin of the material (natural or synthetic), the specific conditions used for the preparation of synthetic carbonates, among other factors, also influence the surface properties of the final material. The acid-base properties have also been determined and again a range of results has been reported [16,19-21]. For instance, some authors reported surfaces with a predominantly Lewis basic character [16,19], while others showed opposite results, 
i.e, that the calcium carbonates surfaces were mainly acidic [20-21]. In the latter case, the interactions with Lewis basic probes such as tetrahydrofurane or ethyl ether were so strong that it was even impossible to quantify their affinity. Once more, this can be highly dependent on the history of the calcium carbonate preparation/origin and preconditioning/measurement temperatures. The effect of the modification of calcium carbonate by stearic acid [11-14,19-20,22-24] and others $[16,18,20,22,25,26]$ has also been assessed by IGC.

The present study aimed at investigating the surface chemical properties of two PCC-silica fillers and comparing them with those of the original PCC. The surface properties of these materials were studied using X-ray photoelectron spectroscopy (XPS) and inverse gas chromatography (at infinite dilution conditions). A range of IGC probes was considered in order to investigate the differences in the surface properties of the studied materials.

\section{Materials and methods}

\section{Samples preparation and characterization}

A commercial scalenohedral PCC was supplied from the industry. The PCC suspension was vigorously stirred and then filtered using a Buckner filter. The solid obtained was oven dried at $105^{\circ} \mathrm{C}$ for $24 \mathrm{~h}$ and ground on a porcelana crucible.

The modification of PCC with silica was performed using dry PCC, tetraethyl orthosilicate, water, ethanol, and ammonia, as previously described [4-5]. Experiments with an ammonia concentration of 0.1 and $0.3 \mathrm{~mol} \mathrm{dm}^{-3}$ were carried out in order to produce two modified PCCs with distinct contents of silica. In each reaction, 15-20 g of PCC were modified allowing to obtain enough amounts of modified mineral for packing at least two IGC columns. 
The characterization of the materials by FTIR and ${ }^{29}$ Si MAS NMR spectroscopy, Xray diffraction, SEM, and thermogravimetric analysis was already reported elsewhere [4-5]. Based on the thermogravimetric analysis, the amount of silica present in the modified PCCs was estimated to be of ca. 22 and $30 \mathrm{wt} \%$, for the PCC-silica materials obtained with 0.1 and $0.3 \mathrm{~mol} \mathrm{dm}{ }^{-3}$ of ammonia concentration, respectively [5].

In this study, and in order to assess the physico-chemical surface properties, the materials were thoroughly analysed by XPS and IGC.

\section{$X$-ray photoelectron spectroscopy}

The X-ray photoelectron spectra were obtained using a Kratos AXIS Ultra HAS equipment. The analysis was carried out with a monochromatic Al Ka X-ray source (1486.7 eV), operating at $15 \mathrm{kV}(90 \mathrm{~W})$, in fixed analyser transmission mode, with a pass energy of $80 \mathrm{eV}$. Wide scan survey spectra were recorded at take-off angle of $90^{\circ}$ and between $0 \mathrm{eV}$ and $1350 \mathrm{eV}$ binding energy with a step size of $1 \mathrm{eV}$ and a dwell time of 200 ms. High-resolution C1s spectra were obtained with a step size of $0.1 \mathrm{eV}$ and a dwell time of $1500 \mathrm{~ms}$; Ca 2p and Si 2p spectra were obtained with a step size of $0.1 \mathrm{eV}$ and a dwell time of $1000 \mathrm{~ms}$. Peak fitting of the high-resolution spectra was performed using Gaussian-Lorentzian peak shapes and Shirley-type background subtraction. Pellets of $1 \mathrm{~mm}$ thickness were previously prepared for the analysis by pressing the samples at $\approx 40 \mathrm{MPa}$ during $2 \mathrm{~min}$.

\section{Inverse gas chromatography}

The material for IGC analysis was previously compacted through axial compression of the powder. The pressed disc was then sieved between 600 and $300 \mu \mathrm{m}$ to obtain "coarse" granulates, before being packed in the IGC column. Using this procedure it was possible to obtain enough gas flow rate in the IGC column. 
IGC analysis was done using a DANI GC 1000 digital pressure control gas chromatograph equipped with a hydrogen flame ionization detector. Stainless-steel columns, $0.5 \mathrm{~m}$ long and $0.4 \mathrm{~cm}$ inside diameter were washed with acetone and dried before packing. Approximately 3-4 g of PCC or PCC-silica were packed into the gas chromatograph column. The columns were shaped in a smooth " $U$ " to fit the detector/injector geometry of the instrument. The packed columns were conditioned overnight (ca. $16 \mathrm{~h}$ ) at $105{ }^{\circ} \mathrm{C}$, under a helium flow, before any measurements were made. Measurements were typically made at the column temperature of $105^{\circ} \mathrm{C}$, with the injector and detector kept at $180^{\circ} \mathrm{C}$ and $200^{\circ} \mathrm{C}$, respectively. For PCC, measurements were also made at $60^{\circ} \mathrm{C}$ as well. Helium was used as carrier gas with a flow rate of $c a$. $15 \mathrm{~mL} / \mathrm{min}$. Small quantities of probe vapor $(<1 \mu \mathrm{L})$ were injected into the carrier gas, allowing work under infinite dilution conditions. The probes used for the IGC data collection were $n$-pentane (C5), n-hexane (C6), n-heptane (C7), n-octane (C8), dichloromethane (DCM), trichloromethane (TCM), 1-pentene, 1-hexene and 1-heptene. Tetrahydrofurane, ethyl ether, ethyl acetate, and acetone were also tested but could not be measured because of strong interaction of these probes with the studied materials. All probes were of chromatographic grade and were used as received (Sigma-Aldrich). Methane was used as the reference probe. The retention times were the average of three injections and were determined by the Conder and Young method [27]. Two different columns were run for each material and the results averaged.

\section{IGC theory}

The principles and details of the IGC theory at infinite dilution conditions can be found elsewhere [7-9]. Briefly, by the Schultz and Lavielle approach [28], the dispersive component of the surface energy of the analysed material $\left(\gamma_{\mathrm{s}}^{\mathrm{d}}\right)$ may be 
estimated from the slope of the linear fit of $R T \ln \left(V_{\mathrm{n}}\right)$ as a function of $2 N \cdot a\left(\gamma_{1}^{\mathrm{d}}\right)^{0.5}$, using the IGC data obtained with a series of apolar probes, usually $n$-alkanes (Eq. 1). In this equation, $R$ is the gas constant, $T$ is the column absolute temperature, $V_{\mathrm{n}}$ is the net retention volume of the probe (calculated from the probe retention time), $N$ is the Avogadro number, $a$ is the molecular surface area of the probe, $\gamma_{\mathrm{s}}^{\mathrm{d}}$ and $\gamma_{1}^{\mathrm{d}}$ are the dispersive components of the surface free energy of the interacting solid material and probe, respectively, and $K$ is a constant that is dependent on the chosen reference state.

$$
R T \ln \left(V_{\mathrm{n}}\right)=\sqrt{\gamma_{\mathrm{s}}^{\mathrm{d}}} 2 N \cdot a \sqrt{\gamma_{1}^{\mathrm{d}}}+K
$$

By the Dorris and Gray approach [29], the $\gamma_{s}^{d}$ can be determined from the difference in the free energy of adsorption due to the introduction of an additional $-\mathrm{CH}_{2}$ - group into the carbon chain of a $n$-alkane probe (Eq. 2). $a_{\mathrm{CH} 2}$ is the molecular area of the $\mathrm{CH}_{2}$ - group, which is usually taken as $0.06 \mathrm{~nm}^{2}$, and $\gamma_{\mathrm{CH} 2}$ is the surface energy of a solid entirely composed of methylene groups (Eq. 3) [9,29]. This approach was used in this work for a better comparison with most of the published results related to PCC.

$$
\begin{aligned}
& \gamma_{\mathrm{s}}^{\mathrm{d}}=\frac{\left[R T \ln \frac{V_{\mathrm{n}}^{\left(\mathrm{C}_{\mathrm{n}+1} \mathrm{H}_{2 \mathrm{n}+4}\right)}}{V_{\mathrm{n}}^{\left(\mathrm{C}_{\mathrm{n}} \mathrm{H}_{2 n+2}\right)}}\right]^{2}}{4 N^{2}\left(a_{\mathrm{CH}_{2}}\right)^{2} \gamma_{\mathrm{CH}_{2}}} \\
& \gamma_{\mathrm{CH}_{2}}=35.6+0.058(293.15-T)
\end{aligned}
$$

For the polar probes, there is a corresponding specific component contribution, $\Delta G_{\mathrm{a}}^{\mathrm{s}}$, in addition to the dispersive component, to the overall free energy of adsorption [9]. This parameter $\left(\Delta G_{\mathrm{a}}^{\mathrm{S}}\right)$ can be estimated by calculating the difference between the experimental value of $R T \ln \left(V_{\mathrm{n}}\right)$ obtained for the polar probe and the corresponding estimation for the equivalent apolar probe (Eq. 4) based on the linear fitting of $R T \ln \left(V_{\mathrm{n}}\right)$ 
vs. $2 N \cdot a\left(\gamma_{1}^{\mathrm{d}}\right)^{0.5}$ for $n$-alkanes (reference line). The reference line may also be obtained by plotting the $R T \ln \left(V_{\mathrm{n}}\right) v s$. the number of carbon atoms.

$$
\Delta G_{\mathrm{a}}^{\mathrm{s}}=-R T \ln \frac{V_{\mathrm{n}}}{V_{\mathrm{n}, \mathrm{ref}}}
$$

\section{Results and discussion}

General characterization of the PCC-silica fillers

The synthesis and characterization of the bulk properties of silica modified PCC fillers was thoroughly described in previous papers [4-5]. Briefly, silica is deposited on the surface of calcium carbonate particles by performing hydrolysis/condensation reactions of TEOS in ethanol/water solutions in the presence of solid PCC, using ammonia as catalyst. FTIR, ${ }^{29} \mathrm{Si}$ MAS NMR, SEM-EDX, and thermogravimetric analysis show the presence of a highly branched silica framework, mainly as a dense film, on the surface of PCC.

As mentioned, reactions were carried out with different ammonia concentrations $\left(0.1\right.$ and $\left.0.3 \mathrm{~mol} \mathrm{dm}^{-3}\right)$ and two PCC-based materials with silica average amounts of 22 and $30 \mathrm{wt} \%$ (determination based on the thermogravimetric analysis [5]) were obtained.

Surface chemical properties of the PCC-silica fillers assessed by X-ray photoelectron spectroscopy

The two samples of PCC-silica filler and the original PCC were analysed by X-ray photoelectron spectroscopy, which, similarly to inverse gas chromatography, is a surface specific technique (up to approximately $10 \mathrm{~nm}$ depth). The XPS results are summarized in Table 1. As expected, $\mathrm{C}, \mathrm{O}$, and $\mathrm{Ca}$ were found as major elements and 
also Si on the surface of the PCC-silica fillers. The atomic percentages of carbon and calcium decreased significantly with the calcium carbonate modification by silica. The $\mathrm{Si} / \mathrm{Ca}$ atomic ratio increased from PCC-silica 22 to PCC-silica 30 confirming the presence of a higher amount of silica in the latter material. High-resolution XP spectra (Fig. 1) showed several components in the region of carbon binding energies, two major signals in the region of calcium binding energies, and one signal in the region of silicon binding energies. In detail, the $\mathrm{C} 1 \mathrm{~s}$ spectra showed two major signals at $285.0 \mathrm{eV}$ and $289.7 \mathrm{eV}( \pm 0.1 \mathrm{eV})$ due to adventitious aliphatic carbon $(\mathrm{C}-\mathrm{H} ; \mathrm{C}-\mathrm{C})$ and carbonate, respectively [30]. Signals of significantly lower intensity were also fitted to the spectra, namely at ca. $286.3 \mathrm{eV}$ and $287.7 \mathrm{eV}$, attributed to carbon in $\mathrm{C}-\mathrm{O}$ bonds, which may arise from impurities present at the surface of PCC [3]. The signal due to carbonate had the highest relative intensity for PCC, as expected, and was less pronounced for PCCsilica 30. Regarding the Ca 2p XP spectra, two distinct lines were clearly fitted for PCC and PCC-silica fillers. Finally, in the Si 2p XP spectra one signal was observed for both PCC-silica fillers. Overall, the results confirm that the surface of PCC-silica materials is enriched with silicon, being present a higher relative amount in PCC-silica 30.

Please, insert Table 1

Please, insert Figure 1

\section{Dispersive component of the surface free energy}

The dispersive component of the surface energy $\left(\gamma_{\mathrm{s}}^{\mathrm{d}}\right)$ of PCC-silica fillers and PCC was determined by the Schultz and Lavielle and the Dorris and Gray methods, using the retention time data measured with the $\mathrm{C} 5$ to $\mathrm{C} 8 n$-alkanes series (Figs. 2A and 2B, respectively). The results are shown in Table 2 . At $105^{\circ} \mathrm{C}$, the values obtained by the Dorris and Gray method were higher by about 5-6 $\mathrm{mJ} \mathrm{m}^{-2}$ than those obtained using the Schultz and Lavielle method. However, for a lower temperature $\left(60{ }^{\circ} \mathrm{C}\right)$ the difference 
between the two methods was reduced. Note that different values of $\gamma_{\mathrm{CH} 2}$ were considered for the calculation of the $\gamma_{\mathrm{s}}^{\mathrm{d}}$ parameter at $60{ }^{\circ} \mathrm{C}$ and $105{ }^{\circ} \mathrm{C}$ (Eq. 2), respectively, 33.3 and $30.7 \mathrm{~mJ} \mathrm{~m}^{-2}$ (Eq. 3).

The values here obtained for $\gamma_{\mathrm{s}}^{\mathrm{d}}$ of PCC are in agreement with previous results of other authors who reported Dorris and Gray values for precipitated calcium carbonate in the $40-60 \mathrm{~mJ} \mathrm{~m}^{-2}$ range $[12,17,19,20]$ at $100{ }^{\circ} \mathrm{C}$. For the dispersive component of PCC determined by the Schultz and Lavielle method, a slight decrease was found when increasing the measurement temperature from $60{ }^{\circ} \mathrm{C}$ to $105^{\circ} \mathrm{C}$ (Table 2). However, the temperature seemed not to affect this parameter when using the Dorris and Gray approach.

The $\gamma_{\mathrm{s}}^{\mathrm{d}}$ value at $105^{\circ} \mathrm{C}$ of the silica-modified PCC filler with a higher silica content (PCC-silica 30) was lower than of the original PCC (Table 2), indicating that the potential of the highest-energy sites of the surface of PCC to undergo Lifshitz-Van der Waals forces can be somewhat decreased by the modification with silica. Since the silica, coating the PCC crystals surface, is amorphous, as demonstrated previously by X-ray diffraction [4], a lower value of $\gamma_{s}^{d}$ is not surprising. In fact, $\gamma_{s}^{d}$ values as low as $20 \mathrm{~mJ} \mathrm{~m}^{-2}$ [25] were obtained by coating calcium carbonate with amorphous silica produced by other methods, while crystalline silica may lead to $\gamma_{\mathrm{s}}^{\mathrm{d}}$ values higher than $100 \mathrm{~mJ} \mathrm{~m}^{-2}$ [25]. However, for the PCC-silica with the lower silica content (PCC-silica 22) no significant differences (relatively to PCC) were found (Table 2). This result may indicate that a certain amount of amorphous silica on the surface of PCC is required to decrease the dispersive component of the surface energy of carbonate.

Please, insert Figure 2

Please, insert Table 2 


\section{Specific interaction parameters with polar probes}

The specific component of the free energy of adsorption $\left(-\Delta G_{\mathrm{a}}{ }^{\mathrm{s}}\right)$ of several Lewis acidic and Lewis basic probes on the surface of the fillers was evaluated. Using the Schultz and Lavielle approach (Fig. 2A) the $-\Delta G_{\mathrm{a}}$ s values of TCM and DCM (as Lewis acids) and the corresponding specific components of the works of adhesion, $W_{\mathrm{a}}^{\mathrm{s}}$, were obtained. The $-\Delta G_{\mathrm{a}}^{\mathrm{s}}$ values of the 1-alkenes (as Lewis bases) were preferentially assessed by the Dorris and Gray approach (Fig. 2B). The results of the specific interactions are shown in Table 3 and Figure 3. The interactions with strong Lewis basic or Lewis amphoteric probes such as tetrahydrofurane and ethyl ether or ethyl acetate and acetone could not be measured because these were retained too strongly in the materials, as a consequence of the high Lewis acidic character of all the materials surfaces. A similar behaviour has been previously observed for calcium carbonates [2021].

\section{Please, insert Table 3}

Concerning the typical Lewis acidic probes, the specific interaction with TCM $\left(W_{\mathrm{a}}^{\mathrm{s}}\right)$ was significantly lower for the silica-modified PCCs in comparison to the original PCC (less than a half, Table 3). However, for DCM, which is also a Lewis acidic probe, an opposite trend has been noted: in fact, due to strong retention, corresponding to strong interactions, the specific interaction with the PCC-silica materials could not be measured, contrary to that with PCC. Although TCM and DCM possess similar electron acceptor properties (i.e., similar electron acceptor number $\left(\mathrm{AN}^{*}\right)$ and a electron donor number (DN) of zero) $[7,8]$, the molecular surface area of DCM molecules is much smaller than that of TCM ones. Thus, other effects rather than simply Lewis acid-base interactions may influence the measured specific interactions with these probes. The less bulky DCM molecules afforded significantly higher specific interaction than the 
TCM with PCC (Table 3). Therefore, it is possible that for the PCC-silica materials, the DCM molecules may be physically entrapped inside the "nanoporous" silica network formed at the PCC surface, thus giving an irreversible interaction (bulk and surface sorption). Besides, for the PCC-silica materials, any chemical reaction between the DCM molecules and the siloxane groups is not to be excluded. Reactions of mineral surfaces with Lewis acidic probes have been reported previously for, e.g., synthetic hydroxyapatite [31].

It was reported for a range of metakaolinitic (and kaolinitic) lamellar materials that the specific interaction with TCM $\left(W_{\mathrm{a}}^{\mathrm{s}}\right)$ varied approximately in the inverse order of the dispersive component of the surface energy $\left(\gamma_{\mathrm{s}}^{\mathrm{d}}\right)$. This was interpreted as being due to variations of roughness at the molecular scale of the materials [32]. However, it is unlikely that this can be translated to the present case, since the $\gamma_{\mathrm{s}}^{\mathrm{d}}$ and $W_{\mathrm{a}}^{\mathrm{s}}$ (TCM) values do not seem to vary in the inverse proportion.

In addition, regarding the specific interactions of TCM with PCC-silica fillers, a higher value was obtained for the PCC-silica with the higher silica content (PCC-silica 30 vs. PCC-silica 22). This may reflect a different relative accessibility of the acidic silanol $(\mathrm{Si}-\mathrm{OH})$ and the basic $\mathrm{Si}-\mathrm{O}-\mathrm{Si}$ groups on the materials surface for the interaction with the Lewis acid-base probes. The presence of these distinct silica structural moieties was previously shown by ${ }^{29} \mathrm{Si}$ MAS NMR spectroscopy [4]. A lower accessibility of the acidic silanol groups relatively to the basic Si-O-Si groups in the PCC-silica 30 particles surface will generate a higher specific affinity with TCM. A different amount of adsorbed water on the materials surfaces, even considering that both samples were subjected to the same temperature and time of pre-conditioning $\left(105^{\circ} \mathrm{C}\right.$ and $\left.16 \mathrm{~h}\right)$, may also influence their acid-base properties: a higher amount of water on the surface of the PCC-silica materials (most probably for PCC-silica 30), may reduce Lewis acidity and 
increase Lewis basicity of the material, by inactivating the highest-energy silanol groups. Overall, it is thus proposed that the surface of PCC-silica 30 presents a more pronounced Lewis basic character.

As mentioned, the interactions of the materials surface with strong Lewis basic probes such as tetrahydrofurane and ethyl ether could not be measured. An attempt to overcome this drawback and measure the Lewis acidic character of the materials surface was done by injecting into the IGC column weak Lewis bases (1-pentene, 1-hexene and 1-heptene). The results of the specific interactions calculated based on the difference of the retention volumes of these bases and those of the corresponding $n$-alkanes with the same number of carbon atoms (Fig. 2B) are shown in Figure 3. Specific interactions with 1-alkenes were in the range of 3-7 $\mathrm{kJ} \mathrm{mol}^{-1}$. For the PCC-silica materials, specific $\pi$ interactions varied in the order 1-pentene $>>1$-hexene $>1$-heptene, the difference between probes being however more pronounced for PCC-silica 22. A decrease of the $\Delta G_{\mathrm{a}}^{\mathrm{s}}$ values of 1-alkenes with the number of carbon atoms has been reported previously for silica and other mineral materials [21]. Specific interactions with the $\pi$ electron donors were higher for PCC-silica 22 probably due to the higher accessibility/exposure of acidic silanol groups at the surface of this sample (as aforementioned), which are more prone for interaction with the Lewis bases.

\section{Please, insert Figure 3}

\section{Conclusions}

The formation of amorphous silica on the PCC surface by sol-gel method provided a slight decrease of the dispersive component of the surface energy of calcium carbonate crystals and a decrease to less than a half of the specific affinity with Lewis acidic probe (trichloromethane), as measured by inverse gas chromatography. Interestingly, the interaction with dichloromethane, also a Lewis acidic probe but with shorter size than 
trichloromethane, seemed to be higher for the PCC-silica materials vs. PCC. These results revealed that steric and Lewis acid-base effects may be both involved in the interaction of the Lewis acidic probes with the PCC-silica materials. The specific interactions with weak Lewis bases (1-alkenes) also varied from PCC to PCC-silica materials, being found for the latter a decreasing trend with the increase of the number of carbon atoms. All the materials proved to have a surface with a dominant Lewis acidic character.

When comparing PCC-silica materials with different levels of silica content (30 wt $\%$ vs. $22 \mathrm{wt} \%$ ), a lower dispersive component of the surface energy, a higher specific interaction with Lewis acidic probe (trichloromethane) and a lower specific interaction with $\pi$ electron donor bases was found for the material with a higher amount of silica. These different results can be probably due to an effect of different accessibility of silanol groups at the materials surface for the interaction with the probes. A higher prevalence of these groups provides a higher Lewis acidity and lower Lewis basicity of the material surface.

In the context of the fillers performance in papermaking, it is proposed that the better compatibility of PCC-silica vs. PCC with cellulose fibres in the handsheets formation may be partially influenced by the Lewis acid-base interactions between the fibres and the modified fillers. However, the hydrogen bonding between the silanol groups of silica and hydroxyl groups of cellulose [5-6] should still represent the main effect. Unfortunately, it was not possible to discriminate the hydrogen bonding specific interactions by inverse gas chromatography. The results presented in this work may be useful as well to predict the compatibility of the new fillers with distinct matrices other than pulp fibres. 


\section{Acknowledgments}

The authors thank FCT (Fundação para a Ciência e Tecnologia) for the financial support in the framework of the project NEWFILL - New modified fillers for papermaking (PTDC/EQU-EQU/120578/2010).

\section{References}

[1] Y. Zhao, Z. Hu, A. Ragauskas, Y. Deng, Improvement of paper properties using starch-modified precipitated calcium carbonate filler, Tappi J. 4 (2005) 3-7.

[2] J. Shen, Z. Song, X. Qian, W. Liu, Modification of papermaking grade fillers: a brief review, Bioresources 4 (2009) 1190-1209.

[3] A.F. Lourenço, J.A.F. Gamelas, P.J. Ferreira, Precipitated calcium carbonate modified by the layer-by-layer deposition method-Its potential as papermaking filler, Chem. Eng. Res. Des. http://dx.doi.org/10.1016/j.cherd.2015.10.026.

[4] J.A.F Gamelas, A.F. Lourenço, P.J. Ferreira, New modified filler obtained by silica formed by sol-gel method on calcium carbonate, J. Sol-Gel Sci. Technol. 59 (2011), 2531.

[5] A.F. Lourenço, J.A.F. Gamelas, C. Zscherneck, P.J. Ferreira, Evaluation of silicacoated PCC as new modified filler for papermaking, Ind. Eng. Chem. Res. 52 (2013) 5095-5099.

[6] A.F. Lourenço, J.A.F. Gamelas, P.J. Ferreira, Increase of the filler content in papermaking by using a silica-coated PCC filler, Nord. Pulp Paper Res. J. 29 (2014) $240-245$. 
[7] J.M.R.C.A. Santos, J.T. Guthrie, Analysis of interactions in multicomponent polymeric systems: the key-role of inverse gas chromatography, Mat. Sci. Eng. R. 50 (2005) 79-107.

[8] J.A.F. Gamelas, The surface properties of cellulose and lignocellulosic materials assessed by inverse gas chromatography: a review, Cellulose 20 (2013) 2675-2693.

[9] P. Mukhopadhyay, H.P. Schreiber, Aspects of acid-base interactions and use of inverse gas chromatography, Colloids Surf. A 100 (1995) 47-71.

[10] E. Brendlé, E. Papirer, A new topological index for molecular probes used in inverse gas chromatography for the surface nanorugosity evaluation, J. Colloid Interface Sci. 194 (1997) 207-216.

[11] E. Papirer, J. Schultz, C. Turchi, Surface properties of a calcium carbonate filler treated with stearic acid, Eur. Polym. J. 20 (1984) 1155-1158.

[12] P.Schmitt, E. Koerper, J. Schultz, E. Papirer, Characterization, by inverse gas chromatography, of the surface properties of calcium carbonate before and after treatment with stearic acid, Chromatographia 25 (1988), 786-790.

[13] M. Dressler, M. Ciganek, Effect of particle treatment of composite filler on its interaction coefficient, J. Chromat. A. 462 (1989) 155-163.

[14] E. Fekete, B. Pukánszky, A. Tóth, I. Bertóti, Surface modification and characterization of particulate mineral fillers, J. Colloid Interface Sci. 135 (1990) 200208.

[15] H. Balard, E. Papirer, Characterization and modification of fillers for paints and coatings, Progress in Org. Coatings, 22 (1993) 1-17.

[16] A. Lundqvist, L. Odberg, Surface energy characterization of pigment coatings by inverse gas chromatography, J. Pulp Pap. Sci. 23 (1997) J298-303. 
[17] D. S. Keller, P. Luner, Surface energetics of calcium carbonates using inverse gas Chromatography, Colloids Surf. A 161 (2000) 401-415.

[18] M. Shui, Polymer surface modification and characterization of particulate calcium carbonate fillers, Appl. Surf. Sci. 220 (2003) 359-366.

[19] E. Fekete, J. Móczó, B. Pukánszky, Determination of the surface characteristics of particulate fillers by inverse gas chromatography at infinite dilution: a critical approach, J. Colloid Interf. Sci. 269 (2004) 143-152.

[20] G. J. Price, D. M Ansari, Surface modification of calcium carbonates studied by inverse gas chromatography and the effect on mechanical properties of filled polypropylene, Polym. Int. 53 (2004) 430-438.

[21] C. Perruchot, M.M. Chehimi, M. Vaulay, K. Benzarti, Characterisation of the surface thermodynamic properties of cement components by inverse gas chromatography at infinite dilution, Cement and Concrete Research 36 (2006) 305-319. [22] A. Calhoun, E. Chiang, Determination of the surface energetics of surface modified calcium carbonate using inverse gas chromatography, J. Vinyl Addit. Technol. 12 (2006) 174-182.

[23] T. Ahsan, B.A. Colenutt, K.S.W. Sing, Gas chromatography of pure and surfacemodified precipitated calcium carbonate, J. Chrom. A. 479 (1989) 17-25.

[24] T. Ahsan, D.A Taylor, The Influence of Surface Energetics of Calcium Carbonate Minerals on Mineral-Polymer Interaction in Polyolefin Composites, J. Adhesion, 67 (1998) 69-79.

[25] M. Shui, Y. Reng, B. Pu, J. Li, Variation of surface characteristics of silica-coated calcium carbonate, J. Colloid. Interf. Sci. 273 (2004) 205-210,

[26] H. P. Schreiber, J.-M. Viau, A. Fetoui, Z. Den, Some properties of polyethylene compounds with surface-modified fillers, Polym. Eng. Sci. 30 (1990) 263-269. 
[27] D.P. Kamdem, B. Riedl, Inverse gas chromatography of lignocellulosic fibers coated with a thermosetting polymer: use of peak maximum and conder and young methods, J. Colloid Interface Sci. 150 (1992) 507-516.

[28] J. Schultz, L. Lavielle, C. Martin, The role of the interface in carbon fibre-epoxy composites, J. Adhesion 23 (1987) 45-60.

[29] G.M. Dorris, D.G. Gray, Adsorption of n-alkanes at zero surface coverage on cellulose paper and wood fibers, J. Colloid Interface Sci. 77 (1980) 353-362.

[30] Surface Science Western (2014) X-ray photoelectron spectroscopy (XPS) reference pages. http://www.xpsfitting.com.

[31] P.H. Harding, J.C. Berg, G. Panjabi, Identification of a surface phase transition in synthetic hydroxyapatite using inverse gas chromatography, J. Mater. Sci. Lett. 17 (1998) 841-843.

[32] J.A.F. Gamelas, E. Ferraz, F. Rocha, An insight into the surface properties of calcined kaolinitic clays: The grinding effect, Colloids Surf. A 455 (2014) 49-57. 


\section{Figure captions}

Fig. 1 High-resolution XP spectra with peak fitting in the region of carbon (A), calcium (B) and silicon (C) binding energies for PCC, PCC-silica 20 and PCC-silica 30.

Fig. 2 Plots for PCC and PCC-silica materials (at $105{ }^{\circ} \mathrm{C}$ ) of: (A) $R T \ln \left(V_{\mathrm{n}}\right) v s$. $2 N \cdot a\left(\gamma_{1}^{\mathrm{d}}\right)^{0.5}$ for the adsorption of $n$-alkanes, dichloromethane and trichloromethane; (B) $R T \ln \left(V_{\mathrm{n}}\right)$ vs. number of carbon atoms for the adsorption of $n$-alkanes and 1-alkenes.

Fig. 3 Specific interactions $\left(-\Delta G_{\mathrm{a}}{ }^{\mathrm{s}}\right)$ of 1-alkenes on the surface of PCC and PCC-silica fillers (1-heptene was not determined for PCC-silica 22 due to extremely high retention time and peak asymmetry). 

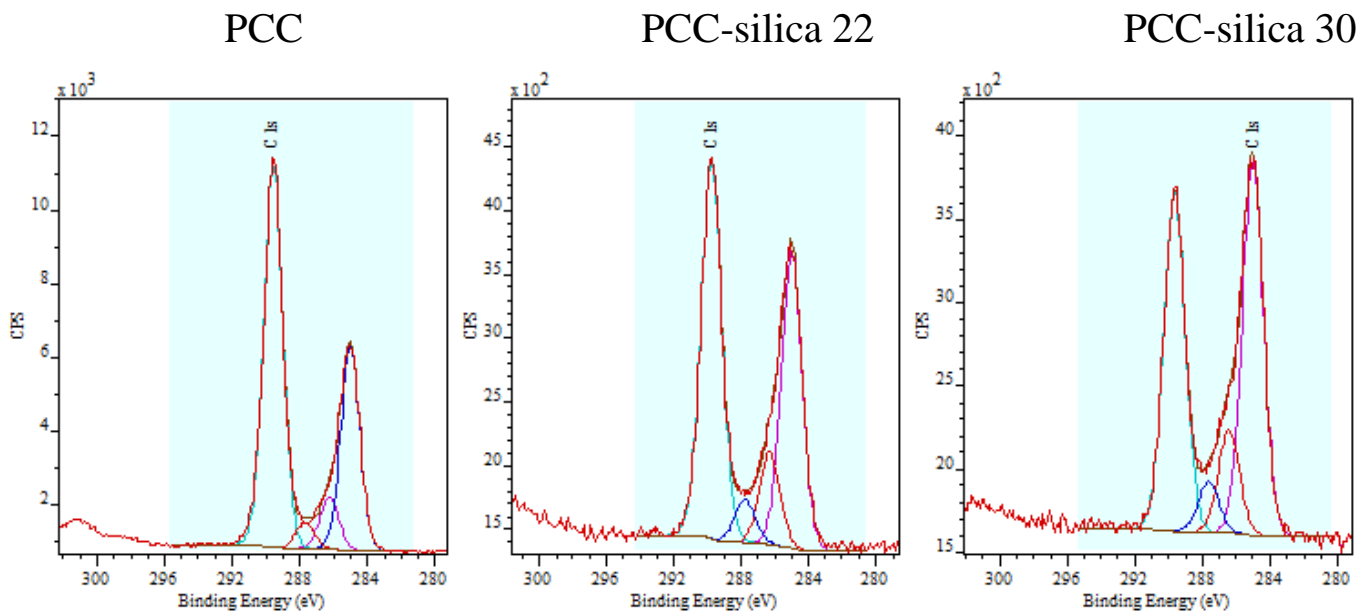

(A)

PCC

PCC-silica 22

PCC-silica 30
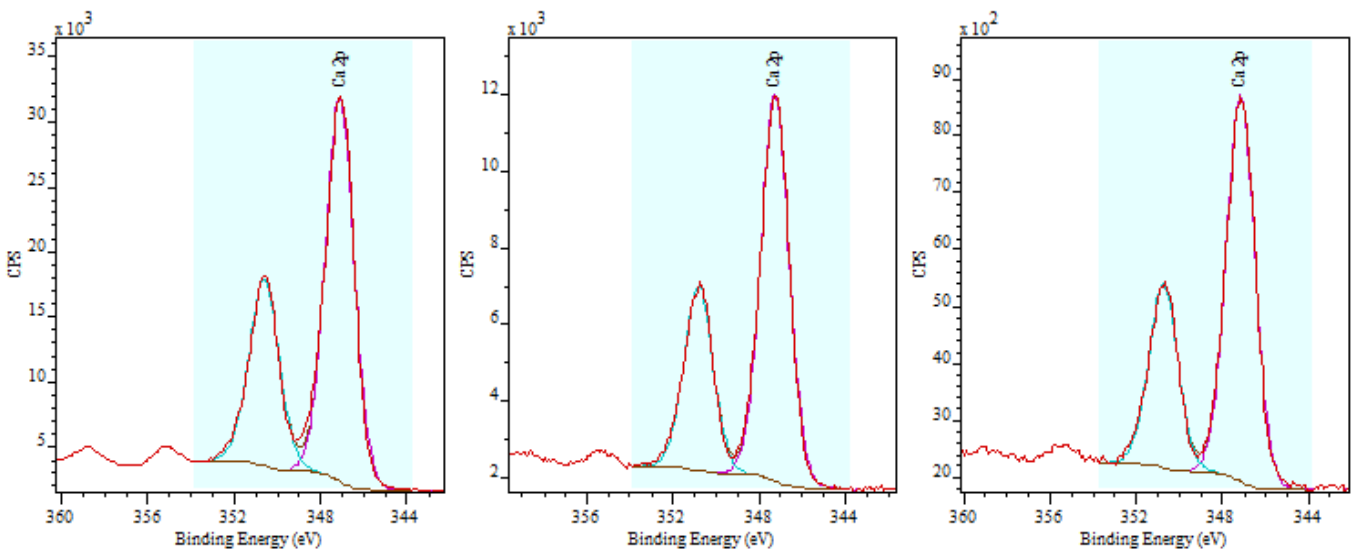

(B)

PCC-silica 22

PCC-silica 30
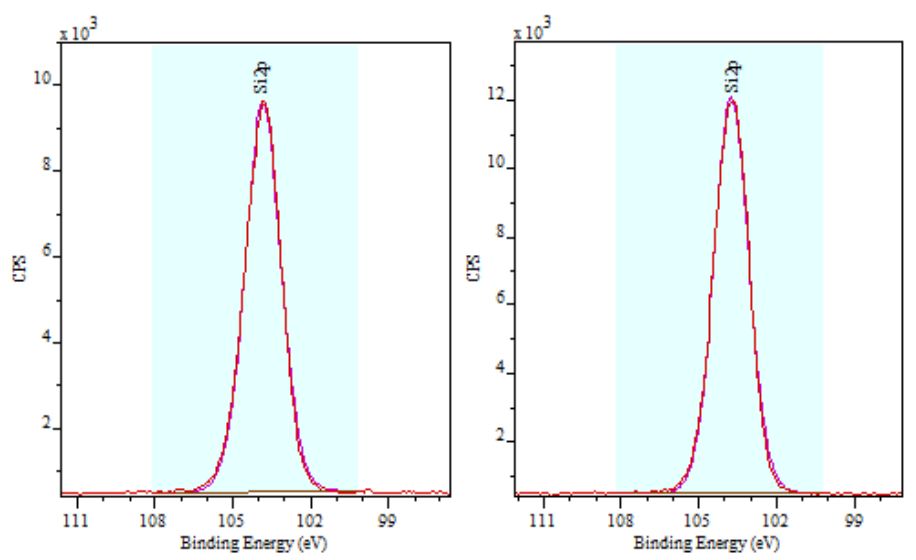

(C)

Fig. 1 
Fig. 2
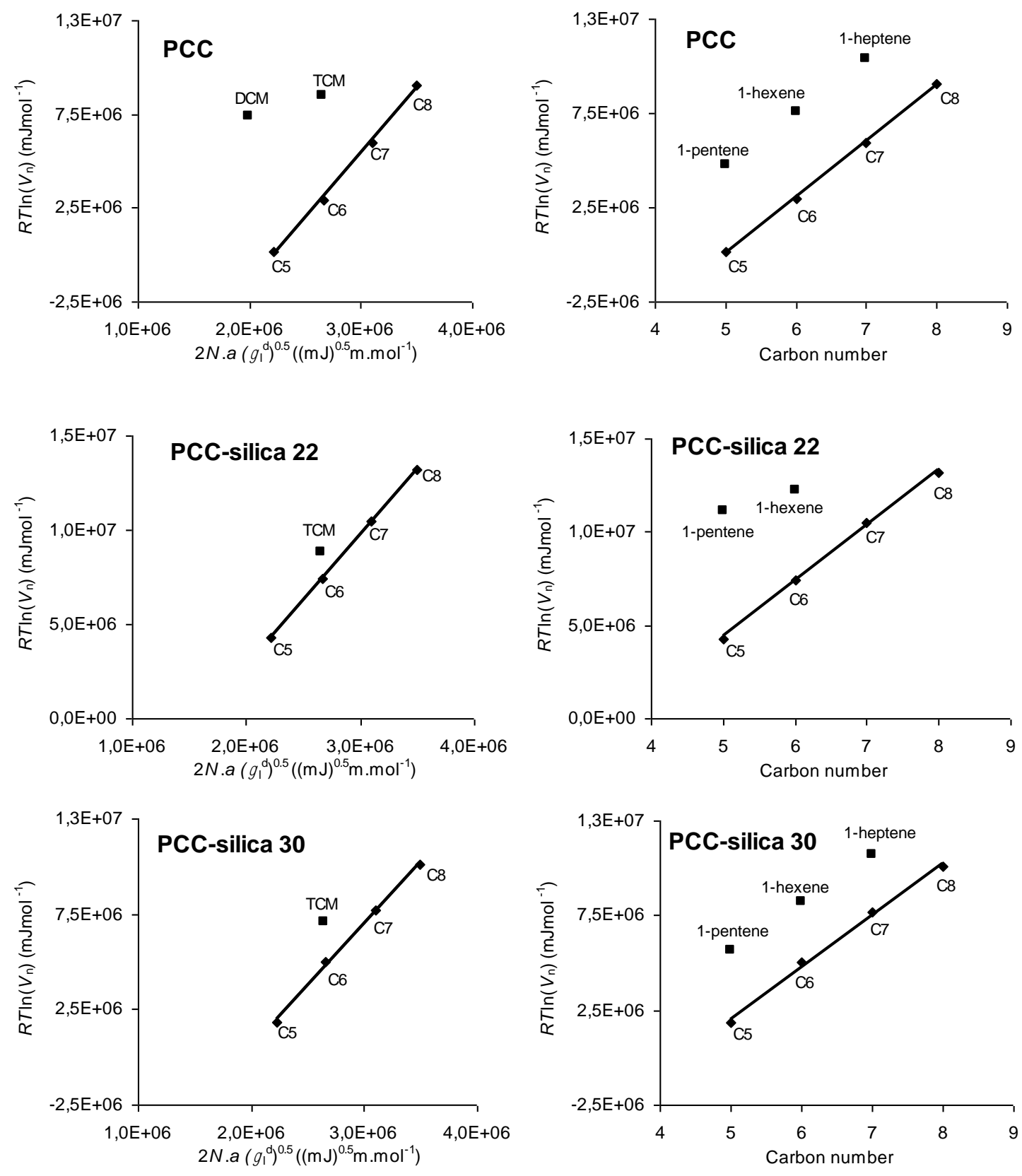

(A)

(B) 
Fig. 3

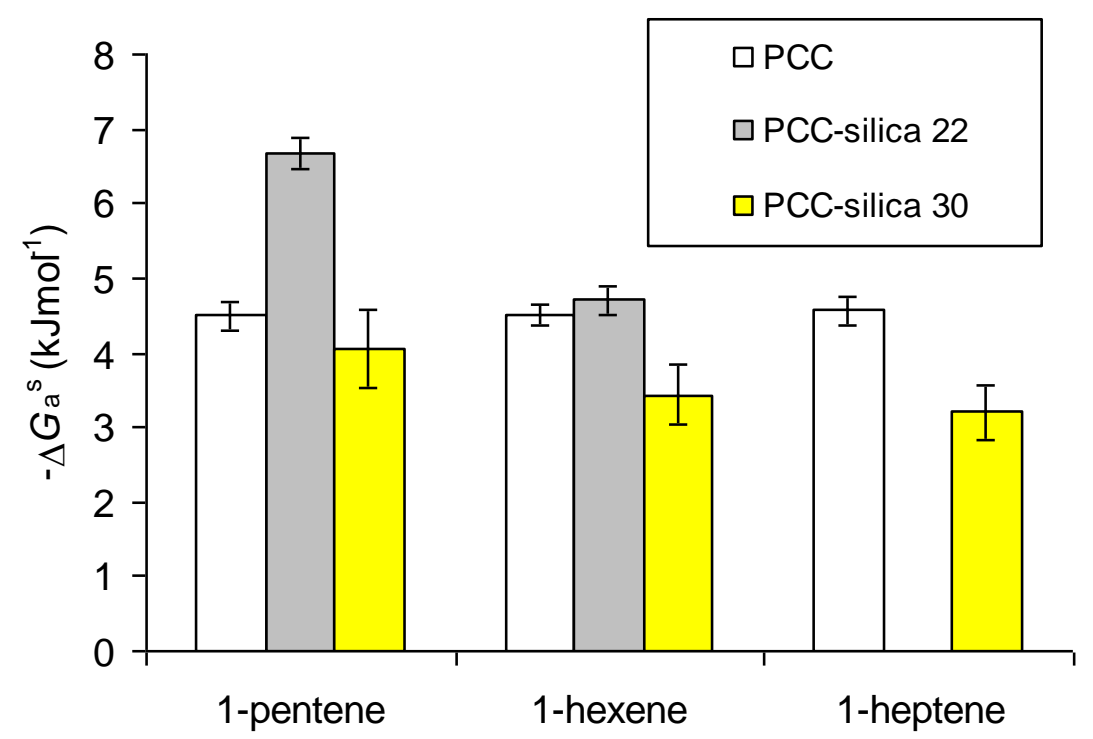


Table 1. Surface chemical analysis (atomic percentage) by XPS of PCC-silica fillers and PCC

\begin{tabular}{cccc}
\hline Element & PCC & ${\text { PCC-silica } 22^{\mathrm{a}}}$ & PCC-silica 30 \\
\hline $\mathrm{C} 1 \mathrm{~s}$ & 33.5 & 13.0 & 10.2 \\
$\mathrm{O} 1 \mathrm{~s}$ & 52.1 & 62.2 & 64.0 \\
$\mathrm{Ca} 2 \mathrm{p} 1+2 \mathrm{p} 3$ & 14.4 & 4.8 & 3.1 \\
$\mathrm{Si}$ & --- & 19.7 & 22.6 \\
$\mathrm{Si} / \mathrm{Ca}$ & 0 & 4.1 & 7.3 \\
\hline
\end{tabular}

${ }^{a}$ low amounts of magnesium were also detected (less than $0.3 \%$ ). 
Table 2. Dispersive component of the surface free energy $\left(\gamma_{\mathrm{s}}^{\mathrm{d}}, \mathrm{mJ} \mathrm{m}^{-2}\right)$ of PCC and PCC-silica fillers

\begin{tabular}{lcc}
\hline Material & $\gamma_{\mathrm{s}}^{\mathrm{d}}$ & $\gamma_{\mathrm{s}}^{\mathrm{d}}$ \\
& $($ Schultz \& Lavielle) & (Dorris \&Gray) \\
\hline PCC $\left(60^{\circ} \mathrm{C}\right)$ & $52.4 \pm 1.1$ & $54.4 \pm 1.1$ \\
PCC $\left(105^{\circ} \mathrm{C}\right)$ & $48.3 \pm 0.9$ & $54.5 \pm 1.1$ \\
PCC-silica $22\left(105^{\circ} \mathrm{C}\right)$ & $49.4 \pm 0.4$ & $55.6 \pm 0.4$ \\
PCC-silica $30\left(105^{\circ} \mathrm{C}\right)$ & $42.9 \pm 2.0$ & $48.3 \pm 2.3$ \\
\hline
\end{tabular}


Table 3. Specific component of the free energy of adsorption $\left(-\Delta G_{\mathrm{a}}^{\mathrm{s}}, \mathrm{kJ} \mathrm{mol}^{-1}\right)$ and work of adhesion $\left(W_{\mathrm{a}}^{\mathrm{s}}, \mathrm{mJ} \mathrm{m}^{-2}\right)$ of Lewis acidic polar probes on the surface of PCC and PCCsilica fillers

\begin{tabular}{l|cc|cc|}
\hline \multirow{2}{*}{ Material } & \multicolumn{4}{|c|}{ Schultz \& Lavielle } \\
\cline { 2 - 5 } & \multicolumn{2}{|c|}{ TCM } & \multicolumn{2}{c|}{ DCM } \\
& $-\Delta G_{\mathrm{a}}^{\mathrm{s}}$ & $W_{\mathrm{a}}^{\mathrm{s}}$ & $-\Delta G_{\mathrm{a}}^{\mathrm{s}}$ & $W_{\mathrm{a}}^{\mathrm{s}}$ \\
\hline PCC $^{\mathrm{a}}$ & $5.48 \pm 0.06$ & $20.7 \pm 0.2$ & $8.72 \pm 0.09$ & $46.0 \pm 0.5$ \\
PCC $^{\mathrm{b}}$ & $5.53 \pm 0.01$ & $20.9 \pm 0.1$ & $9.10 \pm 0.04$ & $48.0 \pm 0.2$ \\
PCC-silica 22 $^{\mathrm{b}}$ & $1.46 \pm 0.08$ & $5.52 \pm 0.32$ & $\mathrm{npd}^{\mathrm{c}}$ & $\mathrm{npd}^{\mathrm{c}}$ \\
PCC-silica 30 $^{\mathrm{b}}$ & $2.42 \pm 0.11$ & $9.14 \pm 0.41$ & $\mathrm{npd}^{\mathrm{c}}$ & $\mathrm{npd}^{\mathrm{c}}$ \\
\hline
\end{tabular}

${ }^{a}$ measurements at $60^{\circ} \mathrm{C} ;{ }^{b}$ measurement at $105^{\circ} \mathrm{C} ;{ }^{\mathrm{c}} \mathrm{npd}$ : not possible to determine due to strong retention of the probe. 